\title{
Consolidation Treatment for Primary Central Nervous System Lymphoma: Which Modality for Whom?
}

\author{
Osnat Bairey $^{a}$ Liat Shargian-Alon $^{a} \quad$ Tali Siegal $^{b}$ \\ anstitute of Hematology, Davidoff Cancer Center, Rabin Medical Center, Petach Tikva and Sackler Faculty of \\ Medicine, Tel Aviv University, Tel Aviv, Israel; ${ }^{b}$ Neuro-Oncology, Davidoff Cancer Center, Rabin Medical Center, \\ Petah Tiqva, Israel
}

\section{Keywords}

CNS lymphoma · High-dose chemotherapy · Autologous stem cell transplantation · High-dose methotrexate .

Consolidation . Whole-brain radiotherapy

\begin{abstract}
Primary central nervous system lymphoma is a rare aggressive disease that largely affects elderly patients and is associated with poor prognosis. The optimal treatment approach is not yet defined and it consists of induction and consolidation phases. The combination of high-dose (HD) methotrexate-based chemotherapy followed by whole-brain radiotherapy (WBRT) prolongs the median progression-free survival (PFS) and overall survival 2- to 3 -fold as compared to WBRT alone but is associated with significant delayed neurotoxicity. Alternative strategies are being investigated in order to improve disease outcomes and spare patients the neurocognitive side effects. These include reduced-dose WBRT, non-myeloablative HD chemotherapy, or HD chemotherapy with autologous stem cell transplantation (HDC/ ASCT). There are no randomized studies that compare all these consolidation regimens head to head but recently HDC/ASCT has been evaluated versus WBRT in prospective randomized studies. These studies proved that WBRT and HDC/ASCT yield similar 2-year PFS with preserved or im-
\end{abstract}

proved cognitive function after HDC/ASCT. Yet, the proportion of patients treated with such intensive consolidation is low, both in real life and in specialized centers, leaving many unsettled issues. This review is appraising current dilemmas related to the choice of consolidating therapeutic modalities, their associated acute and delayed toxicity, and future prospects for alternative approaches in the elderly.

(c) 2020 S. Karger AG, Basel

\section{Introduction}

Primary central nervous system lymphoma (PCNSL) is defined as a unique entity in the WHO classification of non-Hodgkin's lymphoma [1]. It resides exclusively in the central nervous system (CNS) and may affect the brain, cranial and spinal nerves, leptomeninges, spinal cord, and/or intraocular (vitreoretinal) components. All these anatomic structures reside in sanctuary areas behind the blood-brain barrier (BBB) or the blood-retinal barrier, thus escaping exposure to most conventionally dosed chemotherapeutic agents and avoiding control of the host immune system [2,3].

PCNSL is a rare aggressive lymphoma accounting for only $1 \%$ of all cases of lymphoma and up to $4 \%$ of primary brain tumors $[4,5]$. As a result, treatment approach 
has largely evolved from findings of retrospective series and single-arm phase II studies with only a handful of phase III studies comparing different treatment modalities. Consequently, the optimal treatment approach is not yet defined.

PCNSL is almost exclusively classified as diffuse large B-cell lymphoma (DLBCL) and corresponds to the more aggressive type of nongerminal center B-cell-like DLBCL. Without treatment the median survival time is no more than 3 months while first-line treatment often achieves a high-rate of remission. Relapses remain frequent though and only a small proportion of failed patients will benefit from salvage therapy. Therefore, the overall outcome is unsatisfactory $[6,7]$.

Studies assessing prognostic factors have consistently correlated older age and worse performance status with poor outcome $[8,9]$. Patients with PCNSL often present with impaired general condition and poor performance status due to neurological dysfunction and cognitive impairment. Elderly patients account for the majority of PCNSL patients [10], and once they present with clinical deterioration they are often perceived as unable to tolerate aggressive treatment. As an outcome, treatment approach in the elderly often differs from that in the young where the intent of treatment is curative. The price of intensive and toxic treatment necessary to achieve the curative goal may be often unacceptable for the vast majority of the elderly patients leading to a conservative therapeutic approach. This is based on the fact that the elderly are prone to develop severe complications related to highdose (HD) chemotherapy, and they also tend to develop enhanced neurotoxicity following consolidation of firstline treatment with whole-brain radiation therapy (WBRT) [11]. Yet, a subgroup of fit elderly patients may still tolerate the curative approach and may be treated with the same manner as younger PCNSL patients, aiming to obtain complete and durable remission.

In this review we outline main issues related to the efforts to maintain remission in newly diagnosed PCNSL. It includes dilemmas related to the choice of consolidating therapeutic modalities, their associated acute and delayed toxicity, and future prospects for alternative approaches in the elderly.

\section{Treatment of PCNSL}

The management of newly diagnosed PCNSL remains a controversial topic with no consensus as to the optimal treatment regimen. Once the combination of high-dose methotrexate (HD-MTX)-based chemotherapy followed by WBRT proved to prolong the median survival 2- to 3 -fold [12], this regimen replaced the previous standard of WBRT alone [13]. HD-MTX-based chemotherapy in combination with WBRT yielded a median overall survival (OS) of 30-60 months with a 5-year survival rate of $30-50 \%$ [5]. However, the combination of chemoradiation therapy was proved to induce delayed neurotoxicity particularly in patients 60 years and older [14]. Subsequently, a phase III randomized study investigated whether omission of WBRT would affect survival [15]. Newly diagnosed PCNSL patients were treated with HD-MTXbased protocol and those who achieved complete response (CR) were randomized to 45-Gy WBRT or observation while second-line chemotherapy was given to patients with non-CR. Even though patients receiving WBRT had a significantly longer progression-free survival (PFS) of 18 months compared with observation (12 months), no difference in OS was observed [15]. Based on the results of this trial and the high risk of neurotoxicity, many clinicians no longer use WBRT as part of up-front treatment of PCNSL and reserve WBRT as a salvage modality [5]. Currently, treatment of PCNSL usually consists of 2 phases: induction and consolidation $[5,16]$. Recently a maintenance treatment has also been introduced [17].

\section{Induction Phase}

Induction chemotherapy aims to rapidly obtain the highest $\mathrm{CR}$ rate before proceeding to consolidation. It consists of HD-MTX-based polychemotherapy as a backbone with regimens and dose variations according to age, performance status, and comorbidity. In the rituximab era, when a better disease control was obtained for systemic DLBCL, rituximab has been added to all first-line regimens of PCNSL without solid evidence for its ability to cross the $\mathrm{BBB}$ or to add efficacy. In fact, its efficacy has not been confirmed in the phase III HOVON study which randomly assigned 200 newly diagnosed PCNSL patients to receive rituximab in addition to HD-MTX-based induction chemotherapy [18]. A recent systematic review and meta-analysis evaluated the role of rituximab in newly diagnosed PCNSL [19]. Only 2 randomized controlled studies that contained regimens with or without rituximab were found. These studies enrolled 343 patients and the meta-analysis conclusion was that there is no evidence for improvement of OS, but the available evidence suggests, with low certainty, that rituximab may improve PFS.

Various immunochemotherapy induction regimens that used different consolidation treatment reported sim- 
ilar 2-year PFS of 57\% (R-MT protocol: rituximab, HDMTX, and temozolomide) [20, 21], 50-79\% (R-MPV: rituximab, HD-MTX, procarbazine, and vincristine) [22-24], and 61\% (MATRix: HD-MTX, cytarabine, thiotepa, and rituximab) [25] and therefore any one of these regimens is a reasonable choice. Yet, it is self-evident that the various HD-MTX-based induction regimens achieve a low CR rate (27-66\%). It is unquestionable that a higher CR rate is the ultimate goal of first-line treatment. Whether it may be obtained by improving the induction regimens or by utilizing consolidation modalities as part of the up-front treatment remains unknown and it will require large-scale studies.

\section{Consolidation Therapy}

It is given after the induction phase to those patients who achieve CR or partial response (PR) and sometimes even to stable disease (SD). It is still unclear whether consolidation improves survival of the subgroup of eligible patients with the lack of head-to-head comparison but the rate of objective response increased after consolidation $[24,26]$ (Table 1). There are 3 modalities of consolidation: WBRT, non-myeloablative HD chemotherapy, and myeloablative conditioning with autologous stem cell transplantation (ASCT).

Consolidation treatments consisted in the past of WBRT. With accumulating evidence that WBRT that follows the induction chemotherapy is associated with delayed neurotoxicity $[27,28]$. Alternative strategies have been sought in order to improve disease outcomes and spare patients the neurocognitive side effects. These include consolidation with a reduced dose WBRT [23, 29], conventional HD chemotherapy [21], or HD chemotherapy with ASCT (HDC/ASCT) [16, 30]. There are no randomized studies that compare all these alternative consolidation regimens head to head but recently HDC/ ASCT has been evaluated versus WBRT in 2 prospective randomized studies [26, 31].

\section{Consolidation with WBRT}

PCNSL is radio sensitive and therefore WBRT has been considered as standard treatment and was routinely applied to patients with PCNSL in clinical practice [32]. Since PCNSL is a diffusely infiltrating tumor that potentially affects the whole brain, radiotherapy is given as WBRT at a dose of 40-45 Gy in 1.8- to 2.0-Gy fractions. It should be noted that the addition of WBRT consolidation to preceding induction with HD-MTX-based

Consolidation Treatment for Primary

CNS Lymphoma chemotherapy has never been proven to significantly prolong OS. Yet, the combination yielded superior results to those obtained with WBRT alone and became the gold standard for all further evaluations of treatment outcomes [33]. With prolonged patients' survival obtained by the combined treatment, delayed neurotoxicity became evident $[34,35]$. High proportion of patients over 60 years who were treated by both HD-MTX-based chemotherapy and WBRT developed severe cognitive decline over time whereas younger patients suffered from less severe and delayed cognitive dysfunction. The true prevalence of late neurotoxicity is underestimated, particularly in the young, as most clinical trials have inadequate long-term neurocognitive follow-up. Late neurotoxicity is often associated with neuroimaging abnormalities and typically MRI reveals diffuse white matter (WM) disease, ventricular dilatation, and cortical/subcortical atrophy. Autopsy findings show myelin and axonal loss, gliosis, spongiosis, rarefication of WM, small and large vessel disease, and sometimes thrombosis $[35,36]$.

WBRT-induced severe neurotoxicity led to attempts to reduce the risk by either changing WBRT fractionation schedule [37] or by consolidating with reduced-dose WBRT (rdWBRT) (23.4 Gy in 13 fractions) [29, 33]. Hyperfractionation has not shown any advantage in cognitive preservation. As for rdWBRT, initial findings suggested no evidence of cognitive decline except for a reduction of motor speed in 12 patients who remained progression free up to 48 months [23]. The use of rdWBRT is currently being evaluated in a randomized phase II trial which compares HD-MTX, procarbazine, rituximab, and cytarabine (R-MPV-A) with or without rdWBRT in 91 newly diagnosed patients (NCT01399372). Preliminary data recently published in a meeting abstract demonstrated improved 2-year PFS (78 vs. 54\%) with no significant increase in neurotoxicity as per investigator's assessment. Further neuropsychological testing and neuroimaging analysis are ongoing to characterize neurocognitive decline and how it compares to other consolidation treatments [29].

With further attempts to reduce the risk of treatmentinduced neurocognitive decline 2 prospective randomized studies compared standard WBRT consolidation (36 or $40 \mathrm{~Gy}$ ) to HDC/ASCT and found that both modalities were effective with similar outcome of 2-year PFS [26, 31]. Prospective neurocognitive evaluation verified that WBRT was associated with more prominent neurocognitive dysfunction compared to the alternative of $\mathrm{HDC} /$ ASCT. 


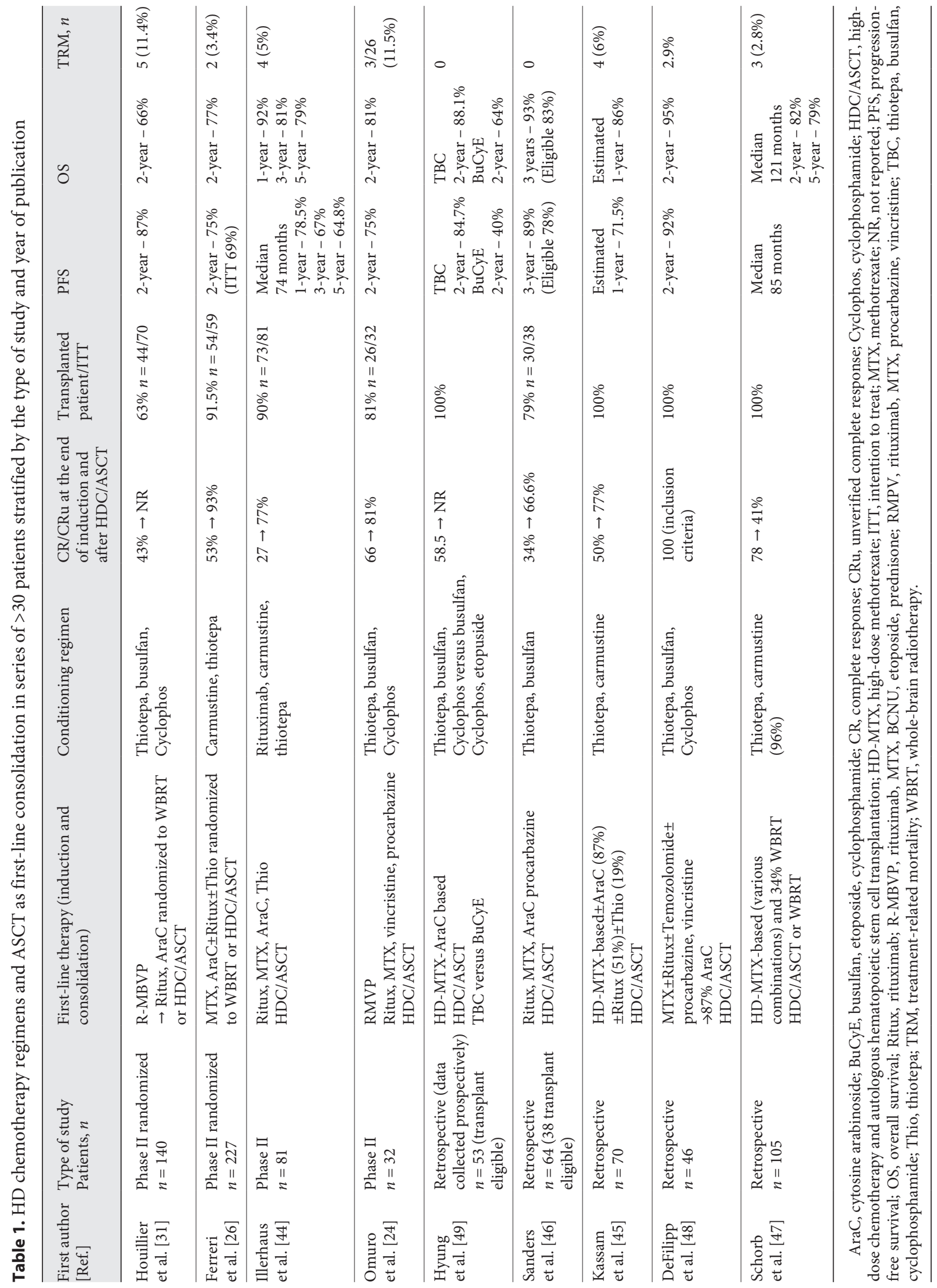


Currently we are avoiding consolidating with WBRT at standard doses due to the foreseen outcome of cognitive dysfunction. However, once the results of rdWBRT study will be matured it may change current clinical preferences, particularly for elderly PCNSL patients for whom the alternative of HD chemotherapy is tricky.

\section{Consolidation with Non-Myeloablative HD Chemotherapy}

Consolidation regimens with HD chemotherapy face major obstacles in the large portion of elderly PCNSL patients whose advanced age and poor general conditions may hamper the ability to use it routinely. Consequently, such consolidation is administered to selected fit patients. Cytarabine has been the main agent that served for consolidation in the elderly [11]. One study retrospectively evaluated whether intensified consolidation with three 28 -day cycles of cytarabine $\left(3 \mathrm{~g} / \mathrm{m}^{2} \mathrm{D} 1-\right.$ 2) improved outcome in patients older than 60 years [38]. Ninety patients were included. They received the MPV induction regimen that was followed by the intensified cytarabine (MPV-AAA). Outcomes were compared with the historical single cytarabine cycle (MPVA). The conclusion was that intensified cytarabine regimen does not improve the efficacy in the elderly but it does increase toxicity. Notably, the rate of treatmentrelated death mounted to $14.4 \%$. Another phase II study used intensive chemotherapy protocol that included consolidation with HD etoposide plus cytarabine that followed an induction with rituximab, HD-MTX, and temozolomide $[21,39]$. The study accrued 44 patients of all ages with 23 of them aged $>60$ years. The OR was $72 \%$, CR 47\%, median PFS was 48 months, and 4-year OS was $65 \%$. The conclusion was that dose-intensive consolidation is feasible and yields rates of PFS and OS at least comparable to those regimens involving WBRT. It is mentioned that patients aged $>60$ years did as well as younger patients but the exact figures of median PFS and OS of the older subgroup are not specified. The CALGB group is conducting a randomized phase II study (NCT01511562) in which patients receive RMT induction followed by consolidation with either HD etoposide-cytarabine or thiotepa-carmustine conditioning followed by ASCT [30]. Preliminary results of the induction phase were published in a meeting abstract and showed that $33 \%$ of the 108 eligible patients did not proceed to consolidation which supports the need to develop more effective induction strategies.

Consolidation Treatment for Primary

CNS Lymphoma

\section{Consolidation with HDC/ASCT}

The purpose of ASCT is to allow the delivery of high doses of chemotherapy. The use of HDC/ASCT in PCNSL is based on its efficacy in systemic lymphomas as is the case in recurrent chemosensitive systemic DLBCL and other aggressive lymphomas where it is applied at the upfront setting in first CR ( $\mathrm{T}$ cell and mantle cell lympho$\mathrm{ma}$ ). The rationale of performing HDC/ASCT in PCNSL is also resting on the fact that in order to penetrate the $\mathrm{BBB}$, most cytotoxic hydrophilic drugs must be administered at high doses [3], a goal best achieved by the conditioning treatment given before ASCT. Furthermore, HDC/ASCT allows to expose the tumor to a number of noncross resistant drugs that are not used in the induction regimen. This increases the possibility to eliminate residual lymphoma cells that may be resistant to the drugs used for induction [16].

Since 2001, various centers have reported their experience with HDC/ASCT in CNS lymphoma [16, 40-42]. These reports contained small numbers of patients (up to 30 patients) with a relatively young median age of 52-56 years and showed that this approach is feasible and probably promising. With accumulating experience and larger number of patients that have been treated with HDC/ ASCT consolidation at first-line setting or at relapse, this approach became recommended by several guidelines as one of multiple options for PSNCL consolidation $[6,32$, 43] including the National Comprehensive Cancer Network (NCCN) guidelines for routine practice for PCNSL. Table 1 describes those studies that reported on HDC/ ASCT of $>30$ patients. These studies include phase II studies, 2 phase II randomized studies, and several large retrospective studies [24, 26, 31, 44-49]. Overall high remission rates and excellent long-term outcomes have been reported with approximately a 2-year PFS of 75$87 \%$ and a 2 -year OS of $66-93 \%$ with a treatment-related mortality (TRM) of $0-11.5 \%$.

Although the above-mentioned guidelines recommend HDC/ASCT as an optional consolidation for PCNSL, several issues stay open:

1 Which is the best induction regimen and is intensifica-

tion needed in order to achieve minimal disease prior to HDC/ASCT?

Prospective as well as retrospective trials reporting on $\mathrm{HDC} / \mathrm{ASCT}$ in the first-line setting used different variations of HD-MTX-based chemotherapy as induction treatment for PSNCL (Table 1). Overall, the rate of CR obtained by the various induction regimens is disappointingly low ranging between 27 and 58.5\%. The

Acta Haematol 2021;144:389-402

DOI: $10.1159 / 000511208$ 
IELSG32 prospective randomized trial [25] compared 3 induction regimens and showed that patients treated with MATRix had a CR rate of $49 \%$ compared with $23 \%$ of those treated with HD-MTX-cytarabine alone. Notably, the addition of rituximab alone to HD-MTX-cytarabine protocol did not yield a significant increase in the CR rate $(30 \%)$. This trial proved that the thiotepa-containing regimen is the more effective. Other types of protocols used HD-MTX in combination with alkylating agents (such as procarbazine, carmustine, temozolomide) and added sequential intensification with HD cytarabine $[24,32,38$, 50]. These protocols yield CR rates similar to the MATRix regimen (Table 1$)$.

It is unclear how intensive the induction treatment should be before proceeding to HDC/ASCT. It is also unsettled whether intensification treatment should be added to the induction in order to minimize residual disease before the consolidation with HDC/ASCT as it carries the risk of augmented toxicity (hematological toxicity and leukoencephalopathy). A different school of thoughts favors the strategy of short and less toxic induction protocol that reduces potential toxicity and enables earlier HDC/ ASCT consolidation with hope of uncompromised efficacy $[46,51]$. The reasoning behind this approach is the concept that the goal of PCNSL therapy is to optimize the rate of ASCT rather than intensification of the induction treatment as the latter may counteract the ability to proceed to ASCT due to toxicity. Sanders et al. [46] retrospectively reviewed the experience of clinical practice guideline that defined ASCT-eligible patients at diagnosis of PCNSL who received a well-tolerated HD-MTX/cytarabine-based induction which optimized stem cell mobilization and ASCT rates (30 of 38 eligible patients underwent successful ASCT). Another clinical trial [51] that adapts the same concept is intending to accrue ASCTeligible elderly patients (age $>65$ years) who will undergo 2 cycles of induction therapy and those achieving at least SD will proceed for HDC/ASCT.

The impact of the response to treatment (CR vs. PR) which is obtained prior to HDC/ASCT consolidation on the desired end result of durable remission remains an open issue. None of the available studies scrutinized this issue and therefore it is unsettled whether treatment intensification is recommended prior to HDC/ASCT once the maximal response to induction is less than a CR.

2 Is HDC/ASCT consolidation effective or are we selecting the good prognosis patients such as the young, fit, and responding patients?

Table 2 summarizes studies that include information on the fraction of patients treated by any type of consoli- dation and specifically by HDC/ASCT. It shows that despite the fact that the overall response rate to induction therapy is ranging between 53.7 and 90\% [72], the fraction of patients proceeding to receive any consolidation is lower, ranging between 21 and $76.9 \%$ of the total reported cohorts. Furthermore, publications of real-life experience show that consolidation with HDC/ASCT is given to only $6-7.1 \%$ of PCNSL patients $[52,53]$. Preselection of patients by prognostic factors, such as younger age and higher performance status, which is a common prerequisite for clinical trials, increases the rate of consolidation by HDC/ASCT to the range of 38-90\% (Table 2). Notably, the median age of patients who receive the latter consolidation is lower than 60 years in most series, and it is another reflection of subgroup selection. Overall, the above figures indicate that consolidation with HD chemotherapy with or without ASCT is usually given to patients with favorable prognostic indicators who respond to chemotherapy while in real life their fraction is probably no more than $21 \%[52,54]$. This is also consistent with the fact that the elderly account for the majority of PCNSL patients $[10,11]$ and most of them are considered noneligible for ASCT.

Whether HDC/ASCT offers a superior efficacy over observation to patients who are eligible to ASCT remains unsolved as there are no randomized studies that compare eligible patients at first remission who are managed with or without HDC/ASCT, though the rate of objective response increased after consolidation [24, 26]. Currently there are 2 prospective randomized trials that compare consolidation with WBRT versus HDC/ASCT that differ somewhat in design but have similar outcomes $[26,31]$.

The first study is the phase II randomized IELSG32 study that initially addressed the issue of which induction protocol is better by comparing 3 protocols. It showed that the MATRix regimen (HD-MTX, cytarabine, thiotepa, and rituximab) was associated with higher response rate $[25,26]$. The second part of this study [26] randomized responding patients $(\mathrm{CR}, \mathrm{PR}$, and $\mathrm{SD})$ between WBRT and carmustine-thiotepa-conditioned ASCT, the primary end point being 2-year PFS. Of the 227 recruited patients, 118 (51.9\%) were randomly assigned to either WBRT or ASCT. The median age of patients undergoing the second randomization was 57 years with $14 \%$ of patients aged 65-70 years. Both WBRT and ASCT were active as they resulted in a substantial increase in the proportion of patients who achieved a CR. There was no significant difference in 2-year PFS between the 2 groups. The PRECIS trial [31] randomized 140 untreated PCNSL adult patients up to the age of 60 years to receive WBRT 


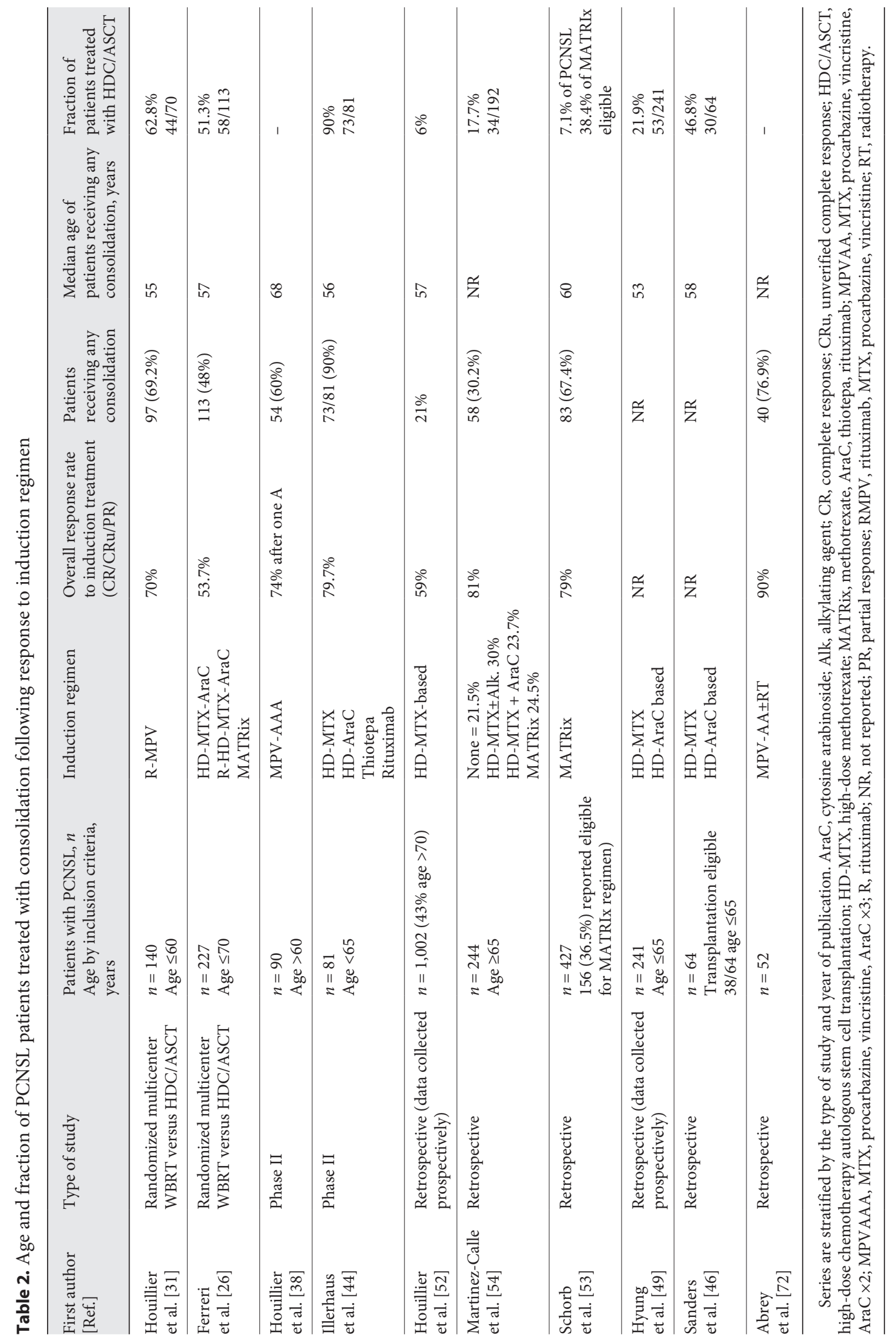


or HDC/ASCT following induction therapy. ASCT conditioning included thiotepa-busulfan-cyclophosphamide, and the primary end point was 2-year PFS. The outcome did not differ significantly between the 2 modalities of consolidation with 2-year PFS for WBRT of $63.2 \%$ (95\% CI 49.5-80.5) versus $86.8 \%$ (95\% CI 76.6-98.3) after HDT/ASCT, with identical 2-year OS between the 2 arms. These 2 randomized studies indicate that the rate of $\mathrm{CR}$ increases following consolidation and support HDC/ ASCT as an alternative to WBRT due to similar efficacy and less neurotoxicity. Yet, TRM should be taken into account when ASCT is offered as consolidation (Table 1).

Two ongoing randomized trials aim to directly compare HDC/ASCT to non-myeloablative HD chemotherapy regimens: a phase II trial of combination of etoposide/ cytarabine versus HDC/ASCT (NCT01511562) and a phase III study of HD-ifosfamide-based combination (DeVIC: dexamethasone, etoposide, ifosfamide, carboplatin) versus HDC/ASCT (NCT02531841).

3 Should HDC/ASCT be offered to all transplant-eligi-

ble patients or just to those responding to induction treatment?

Several studies reported that the rate of CR has increased after HDC/ASCT consolidation (Table 1) [26,31, $44,47]$. The IELSG32 study reported that 24 of 28 patients whose maximal response to induction was PR or SD achieved a CR following HDC/ASCT [26]. Illerhaus et al. [44] treated 73 of 79 patients who completed induction protocol with HDC/ASCT irrespective of their response status. The $\mathrm{CR}$ rates increased from $26.6 \%$ after induction to $77.2 \%$ at 30 days after HDC/ASCT. An ad hoc analysis of possible prognostic effect of remission status (CR vs. no $\mathrm{CR}$ ) before HDC/ASCT on PFS and OS (calculated from the date of HDC/ASCT) did not find any obvious effect [44]. Another retrospective study [47] reported that 7 of 20 patients with progressive disease prior to HDC/ ASCT achieved an ongoing CR without further treatment.

It should be noted that the response criteria for PCNSL include in the definition of unconfirmed CR $(\mathrm{CRu})$ patients with a small but persistent enhancing abnormality on MRI [55]. As it is often difficult to ascertain whether this represents a residual nidus of tumor or scar tissue, the nature of these abnormalities may only be determined by observing the patient with serial scans. So far, FDG PET has limited sensitivity for detecting such small residual lesion in the brain due to high background activity of the normal brain tissue. By contrast, amino acids analogs tracers are more attractive for that purpose due to the low background activity in the brain and in fact a recent study evaluated the utility of $\mathrm{C}^{11}$-methionine for differentiating between $\mathrm{CRu}$ and PR and has defined the tumor-to-normal tissue count in PCNSL [56]. Therefore, CRu is prone for interobserver interpretation as it is not a sharply defined condition (presence or absence of residual macroscopic disease). This may introduce a misinterpretation of the proportion of patients in PR versus CRu between those studies that report on the remission status prior to consolidation. Yet, patients who fail to respond to standard chemotherapy do not necessarily have chemotherapy-resistant disease and may still derive benefit from dose intensified CNS penetrating chemotherapy. However, whether or not remission status before HDC/ASCT is of similar prognostic value as in systemic aggressive lymphoma needs to be investigated in the future.

4 Can HDC/ASCT be offered to elderly patients?

Intensive CNS directed treatment has typically been restricted to younger patients (aged $<65$ years) (Table 2) owing to concerns related to tolerability and treatmentinduced toxicity. Currently, there is an evolving shift from this concept similar to the trend in other hematological malignancies. Multiple myeloma, acute leukemia, and myelodysplastic syndrome are among the leading indications for ASCT and allogeneic hematopoietic stem cell transplantation. However, with an age over 65 years at diagnosis, any fit patient will be transplanted, as transplantation is the ultimate curative option.

With improvements in supportive care, mobilization techniques, and the use of less intensive conditioning regimens, the rate of HSCT among elderly patients has increased markedly in the past 2 decades $[40,52,53]$. ASCT activity in elderly patients ( $\geq 65$ years old) increased from $3.4 \%$ in 2000 to $9.8 \%$ in 2014 . The largest reported experience to date on outcomes of ASCT in elderly patients comes from the European Society for Blood and Marrow Transplantation (EBMT) informing on a total of 21,390 ASCT in patients $\geq 65$ years old treated between 2000 and 2014 [57]. The nonrelapse mortality at 1 year was $4.9 \%$ and 1 - and 3-year OS were 87 and 67\%, respectively. Multivariate analyses showed that performance status had a more significant independent impact on patient outcomes than chronological age. Therefore, age should not be an exclusive criterion per se for HSCT decision but a broader multidimensional assessment including fitness, comorbidities, physiologic reserve, frailty, and socioenviromental circumstances have all to be integrated into the evaluation.

Schorb et al. [58] retrospectively investigated the outcomes of elderly PCNSL patients (age $\geq 65$ years) who underwent intensive treatment with thiotepa-based HDC/ 
ASCT. Of the 52 patients, 15 were treated as part of a firstline approach and 37 as part of a second or subsequent line. The median age was 68.5 years and the median Karnofsky performance status before HDC/ASCT was $80 \%$. The CR rates rose from $34.6 \%$ before HDC/ASCT to 69.2\%. Median PFS was 51.1 months and median OS 122.3 months. The 2 -year PFS and OS were 62 and $70.8 \%$, respectively. TRM was $3.8 \%$. The authors conclude that in selected elderly PCNSL patients, thiotepa-based conditioning for HDC/ASCT seems safe and effective if conducted at experienced centers.

A recent retrospective study reported on outcomes of 244 consecutive PCNSL patients aged 65 years or older who were treated in 14 UK PCNSL-specialized centers [54]. Patients were categorized based on clinicians' treatment choice into palliative $(21.3 \%$, oral chemotherapy or WBRT), less intensive $(30.3 \%, \mathrm{HD}-\mathrm{MTX} \pm$ rituximab + alkylators), and intensive treatment (48.4\%, HD-MTX/ cytarabine combinations). Induction treatment was completed in $47.3 \%$ of patients in the less intensive category, while $70.3 \%$ finished induction in the intensive cohort. Yet, consolidation was given to only $10.8 \%$ of the less intensive cohort and to $42.4 \%$ of the intensive group. HDC/ ASCT was applied as consolidation to 34 of 118 (28.8\%) patients in the intensive cohort and constituted $13.9 \%$ of elderly PCNSL patients referred for treatment at the specialized centers. The CR rate, 2 -year PFS, and OS were 49, 11 , and $24 \%$, respectively, for the less intensive and 69 , 40 , and $50 \%$ for the intensive group. TRM for MTX-treated patients was $6.8 \%$. These retrospective data suggest that a subset of older patients achieve favorable outcomes with HD-MTX-based intensive induction regimen and may benefit from HDC/ASCT consolidation.

An above-mentioned and ongoing phase II study (German clinical trials registry DRKS00011932) is investigating the efficacy of age-adapted induction treatment followed by HDC/ASCT [51]. The study accrues elderly PCNSL patients for treatment with 2 cycles of a modified and less toxic MATRix induction protocol which excludes thiotepa and reserves it for the conditioning protocol of busulfan-thiotepa followed by ASCT. Such prospective studies are clearly needed in order to allow better identification of older PCNSL patients who are likely to benefit from intensive treatment approach. Currently, it is unknown what the upper age limit is for HDC/ASCT and what treatment may serve well as an alternative to either HDC or WBRT consolidation.

5 Which conditioning regimen to use before ASCT?
There are 2 types of conditioning combinations that have been used in patients with PCNSL either in the firstline setting or at relapse [16]:

1 Thiotepa-based regimens - which include combination of thiotepa and BCNU or thiotepa with HD busulfan, with or without cyclophosphamide.

2 Thiotepa-free regimens - which include BEAM (BCNU, etoposide, cytarabine, melphalan), BUCY (busulfan, cyclophosphamide, etoposide), and LEED (cyclophosphamide, etoposide, melphalan, dexamethasone) regimens.

Initial experience with $\mathrm{HDC} / \mathrm{ASCT}$ in PCNSL has been with BEAM conditioning regimen as BEAM is commonly used for patients with systemic lymphoma [40, 59]. This led to disappointing results which are probably the outcome of the limited ability of the agents in BEAM regimen to effectively cross the $\mathrm{BBB}$ [60]. In consequence, BEAM is not recommended as a conditioning regimen for PCNSL. A thorough understanding of the pharmacology of agents used in the conditioning regimen aids in the design and understanding of the preferred protocols. CNS penetration is approximated by measuring CSF level as compared to serum level. This type of evaluation shows low CSF levels for BEAM components such as a 5\% penetration for etoposide, $6-22 \%$ for cytarabine, and $10 \%$ for melphalan. In contrast, busulfan, BCNU, and thiotepa have excellent penetration into the CNS with CSF levels in excess of $80 \%$ serum levels for busulfan and thiotepa, and $50-80 \%$ for BCNU [60]. Thiotepa is a lipophilic drug with high penetration into the CNS and is an important component in the conditioning protocols that are yielding favorable outcomes. Furthermore, it seems that TRM is probably lower in those regimens that combine thiotepa with one additional agent (e.g., carmustine or busulfan) while omitting cyclophosphamide (Table 1) with no obvious compromised outcomes. Apparently, this should be affirmed in prospective randomized clinical trials.

\section{Neurotoxicity Associated with Consolidation}

Neurocognitive dysfunction is defined as a negative change in neurocognitive function that is independent of normal aging and may affect activities of daily living and quality of life (QoL). Whether such an effect is observed in long-term survivors of PCNSL treated with standard chemotherapy only is still unclear.

Delayed neurotoxicity in PCNSL became evident with prolonged patient survival as a result of HD-MTX-based chemotherapy followed by WBRT $[28,33,34]$. The risk 
of neurotoxicity increases with advanced age at treatment and in patients with prolonged disease-free survival [22]. We are not reviewing here radiation-induced neurotoxicity in details as this subject has been extensively covered by previous publications $[28,33,61,62]$. Shortly, the clinical manifestations of radiation-induced neurotoxicity include progressive cognitive dysfunction, ataxia, and urinary incontinence, seizures, and sometimes cerebrovascular events, all of which cause severe disability that eventually may lead to death. Neuroimaging characteristics are progressive WM abnormalities, ventricular dilatation, and cortical atrophy. Histological and animal studies showed that CNS alterations and dysfunction develop much earlier than the initial clinical manifestations that appear at 6 months to 1 year following radiation exposure. The mechanism of radiation-induced neurotoxicity includes acute radiation-triggered CNS inflammation, injury to neuronal lineages, and accessory cells along with their progenitors, and loss of supporting structure integrity. Moreover, these injury-related processes probably alter the signaling microenvironment in progenitor cell niches in the brain and the hippocampus, which is a critical structure to memory and cognition. Progenitor cell niche degradation can cause progressive neuronal loss and cognitive disability.

Modified treatment approaches replace WBRT consolidation by adding HD chemotherapy with or without ASCT aiming to avoid radiation-induced neurotoxicity. But, chemotherapy-related cognitive impairment is a well-recognized clinical consequence of cancer treatment $[63,64]$, and recently a long-term clinically relevant rodent model demonstrated the negative impact of MTX on oligodendrocytes and brain WM which are associated with cognitive impairment [65]. Indeed, WM changes can often be observed on MRI of patients completing induction treatment, even prior to consolidation phase. Long-term survivors of HSCT are at risk for late effects that may adversely affect their QoL [66], and neurocognitive dysfunction can significantly impact the early and late post-HSCT course and may cause substantial posttransplant morbidity $[67,68]$.

Assessment of neurocognitive impairment in PCNSL patients is particularly complicated because most patients have cognitive impairment at diagnosis which is likely related to disease burden and possibly also to the adverse effects of antiepileptic and corticosteroid medications [69]. Therefore, it is not surprising that most studies that include prospective neurocognitive assessments report on improvement in most cognitive domains when referring to baseline evaluation in treatment-responsive
PCNSL patients $[24,26,31,46,69]$. Those studies that evaluated neurocognitive function following HDC/ASCT report on preserved or improved cognitive function after transplantation $[26,31,46,50]$ - unlike the cognitive decline observed after WBRT. However, the prospective evaluation was usually limited to a period of approximately 2 years, while in adult HSCT survivors, an incidence of neurocognitive dysfunction of up to $60 \%$ has been documented at 22-82 months post-transplantation $[70,71]$. Whether delayed neurocognitive impairment may develop in long-term progression-free PCNSL survivors deserves further assessment, but a recent study suggests that this may be the case [69]. In this study cognitive function was prospectively characterized in PCNSL patients with long-term remission after consolidation with rdWBRT or HCT/ASCT. The evaluation was performed at diagnosis, post-induction, and yearly after consolidation up to 5 years. The performance was impaired on most cognitive tests at diagnosis with significant improvement from baseline up to 3 years on several cognitive domains. However, a later decline in attention/executive functions and memory was observed in both groups with delayed increase in brain structure abnormalities. In patients treated with HCT/ASCT, WM abnormalities increased over time, and patients with more WM changes and cortical atrophy had worse cognitive performance regardless of the treatment modality. These observations need further large-scale verification in future studies, but they indicate that delayed neurotoxicity might be associated with HDC/ASCT.

\section{Maintenance Treatment as a Possible Alternative to Consolidation}

Maintenance therapy involves the regular administration of chemotherapy, targeted therapy, or immune-modulating agents. If proved effective, it may serve as an alternative to current consolidation measures in frail patients at high-risk for relapse that cannot tolerate aggressive chemotherapy and particularly in the elderly patients with PCNSL [17]. There are several available and promising chemotherapy and targeted agents that may have a role as maintenance treatment in PCNSL (e.g., procarbazine, temozolomide, lenalidomide, and ibrutinib) but their effectiveness has to be studied in a prospective manner. Maintenance therapy may delay relapses and maintain tumor dormancy when administered as metronomic chemotherapy or targeted therapy. However, owing to the investigational nature of this avenue in PCNSL and the current lack 
of proven efficacy in the up-front setting all maintenance regimens are considered empirical in terms of optimal dose and schedule. Today, there is no randomized clinical trial in PCNSL proving that maintenance is of benefit and therefore it is currently an experimental approach. There are several ongoing clinical trials that evaluate maintenance treatment. An ongoing phase III randomized trial (NCT02313389) that examines the impact of 7 monthly R-MT (HD-MTX, temozolomide, rituximab) maintenance cycles given to elderly patients ( $>60$ years) with PCNSL who achieved a CR. A phase II study evaluates ibrutinib maintenance treatment in elderly patients who respond to induction phase (NCT02623010). A randomized phase II study compares procarbazine and lenalidomide as maintenance in HD-MTX-eligible elderly patients. It also contains an arm of temozolomide maintenance given to HD-MTX-ineligible elderly patients. Another randomized phase II trial compares obinutuzumab maintenance to observation (NCT02498951), and a phase $\mathrm{Ib} / \mathrm{II}$ study evaluates lenalidomide as a maintenance option (NCT0412035). Based on current knowledge, maintenance treatment might be considered in the clinical setting only for elderly or frail patients who cannot tolerate consolidation with intensive chemotherapy or radiation therapy. If possible, these patients should be referred to ongoing clinical trials. Those ongoing studies should cautiously evaluate the risks, benefits, and cost-effectiveness of prolonged maintenance treatment in PCNSL.

\section{Conclusions}

The optimal treatment for PCNSL is an unresolved matter, especially pertaining to up-front consolidation and to the management of elderly patients, who comprise the majority of patients with this infrequent disease. The challenges are numerous. Non-myeloablative HD chemotherapy and HDC/ASCT aim to eradicate residual disease left after induction treatment and are currently used as alternative consolidation modalities to WBRT. These approaches are offered to selected subgroups of responding patients that often exclude the elderly. Yet, a subgroup of fit elderly patients may still tolerate such an aggressive approach, and this is currently a topic of future trials that need to focus on stratification of the elderly with the goal of achieving and maintaining remission. The objectives of future studies should entail aims such as improving tolerability, minimizing treatment-induced toxicity, preserving long-term cognitive function and QoL side by side with the ultimate goal of prolonging PFS and OS. For those patients who are unfit for HD chemotherapy, an alternative approach may be offered by maintenance therapy that should be prospectively examined. Those open issues require multi-institutional and multidisciplinary efforts that will assess the potential of new agents for improving outcome and maintaining QoL. Meanwhile, consolidation treatment should be considered for all patients with responsive disease after induction of chemotherapy. The decision on which consolidation treatment to choose is based on patient age, fitness for HD chemotherapy (performance status, comorbidities), and the patient's wishes. Upcoming results of NCT01511562 phase II study comparing etoposide/cytarabine versus HDC/ASCT and NCT02531841 phase III study comparing DeVIC (dexamethasone, etoposide, ifosfamide, and carboplatin) versus HDC/ASCT will help to decide between non-myeloablative chemotherapy consolidation and HDC/ASCT consolidation. For patients unfit for HD chemotherapy, low-dose irradiation might be an option, and we hope that currently ongoing studies that evaluate maintenance treatment will prove its effectiveness in ameliorating the dismal prognosis of this group of patients.

\section{Conflict of Interest Statement}

Osnat Bairey received an investigator-initiated grant from Janssen.

\section{Funding Sources}

The authors did not receive any funding.

\section{Author Contributions}

Osnat Bairey: manuscript design and implementation, writing, and manuscript review. Liat Shargian-Alon: manuscript review and approval. Tali Siegal: manuscript design and implementation, writing, and manuscript review. 


\section{References}

1 Swerdlow SH, Campo E, Pileri SA, Harris NL, Stein H, Siebert R, et al. The 2016 revision of the World Health Organization classification of lymphoid neoplasms. Blood. 2016;127(20): 2375-90.

2 Daneman R, Prat A. The blood-brain barrier. Cold Spring Harb Perspect Biol. 2015;7(1): a020412.

3 Muldoon LL, Soussain C, Jahnke K, Johanson C, Siegal T, Smith QR, et al. Chemotherapy delivery issues in central nervous system malignancy: a reality check. J Clin Oncol. 2007; 25(16):2295-305.

4 Deckert M, Engert A, Brück W, Ferreri AJ, Finke J, Illerhaus G, et al. Modern concepts in the biology, diagnosis, differential diagnosis and treatment of primary central nervous system lymphoma. Leukemia. 2011;25(12): 1797-807.

5 Grommes C, Rubenstein JL, DeAngelis LM, Ferreri AJM, Batchelor TT. Comprehensive approach to diagnosis and treatment of newly diagnosed primary CNS lymphoma. Neuro Oncol. 2019;21(3):296-305.

6 Fox CP, Phillips EH, Smith J, Linton K, Gallop-Evans E, Hemmaway C, et al. Guidelines for the diagnosis and management of primary central nervous system diffuse large B-cell lymphoma. Br J Haematol. 2019;184(3):34863.

7 Reni M, Mazza E, Foppoli M, Ferreri AJ. Primary central nervous system lymphomas: salvage treatment after failure to high-dose methotrexate. Cancer Lett. 2007;258(2):165-70.

8 Abrey LE, Ben-Porat L, Panageas KS, Yahalom J, Berkey B, Curran W, et al. Primary central nervous system lymphoma: the Memorial Sloan-Kettering Cancer Center prognostic model. J Clin Oncol. 2006;24(36):5711-5.

9 Ferreri AJ, Blay JY, Reni M, Pasini F, Spina M, Ambrosetti A, et al. Prognostic scoring system for primary CNS lymphomas: the International Extranodal Lymphoma Study Group experience. J Clin Oncol. 2003;21(2):266-72.

10 Mendez JS, Ostrom QT, Gittleman H, Kruchko C, DeAngelis LM, Barnholtz-Sloan JS, et al. The elderly left behind-changes in survival trends of primary central nervous system lymphoma over the past 4 decades. Neuro Oncol. 2018;20(5):687-94.

11 Siegal T, Bairey O. Primary CNS lymphoma in the elderly: the challenge. Acta Haematol. 2019;141(3):138-45.

12 DeAngelis LM, Yahalom J, Thaler HT, Kher U. Combined modality therapy for primary CNS lymphoma. J Clin Oncol. 1992;10(4): 635-43.

13 Nelson DF, Martz KL, Bonner H, Nelson JS, Newall J, Kerman HD, et al. Non-Hodgkin's lymphoma of the brain: can high dose, large volume radiation therapy improve survival? Report on a prospective trial by the Radiation Therapy Oncology Group (RTOG): RTOG 8315. Int J Radiat Oncol Biol Phys. 1992; 23(1):9-17.
14 Gavrilovic IT, Hormigo A, Yahalom J, DeAngelis LM, Abrey LE. Long-term follow-up of high-dose methotrexate-based therapy with and without whole brain irradiation for newly diagnosed primary CNS lymphoma. J Clin Oncol. 2006;24(28):4570-4.

15 Thiel E, Korfel A, Martus P, Kanz L, Griesinger F, Rauch M, et al. High-dose methotrexate with or without whole brain radiotherapy for primary CNS lymphoma (G-PCNSL-SG-1): a phase 3, randomised, non-inferiority trial. Lancet Oncol. 2010;11(11):1036-47.

16 Ferreri AJ, Illerhaus G. The role of autologous stem cell transplantation in primary central nervous system lymphoma. Blood. 2016; 127(13):1642-9.

17 Bairey O, Siegal T. The possible role of maintenance treatment for primary central nervous system lymphoma. Blood Rev. 2018 Sep; 32(5):378-86

18 Bromberg JEC, Issa S, Bakunina K, Minnema MC, Seute T, Durian M, et al. Rituximab in patients with primary CNS lymphoma (HOVON 105/ALLG NHL 24): a randomised, open-label, phase 3 intergroup study. Lancet Oncol. 2019;20(2):216-28.

19 Schmitt AM, Herbrand AK, Fox CP, Bakunina $\mathrm{K}$, Bromberg JEC, Cwynarski $\mathrm{K}$, et al. Rituximab in primary central nervous system lymphoma-A systematic review and metaanalysis. Hematol Oncol. 2019;37(5):548-57.

20 Glass J, Won M, Schultz CJ, Brat D, Bartlett $\mathrm{NL}$, Suh JH, et al. Phase I and II study of induction chemotherapy with methotrexate, rituximab, and temozolomide, followed by whole-brain radiotherapy and postirradiation temozolomide for primary CNS lymphoma: NRG oncology RTOG 0227. J Clin Oncol. 2016;34(14):1620-5

21 Rubenstein JL, Hsi ED, Johnson JL, Jung SH, Nakashima MO, Grant B, et al. Intensive chemotherapy and immunotherapy in patients with newly diagnosed primary CNS lymphoma: CALGB 50202 (Alliance 50202). J Clin Oncol. 2013;31(25):3061-8.

22 DeAngelis LM, Seiferheld W, Schold SC, Fisher B, Schultz CJ; Radiation Therapy Oncology Group S. Combination chemotherapy and radiotherapy for primary central nervous system lymphoma: Radiation Therapy Oncology Group Study 93-10. J Clin Oncol. 2002; 20(24):4643-8.

23 Morris PG, Correa DD, Yahalom J, Raizer JJ, Schiff D, Grant B, et al. Rituximab, methotrexate, procarbazine, and vincristine followed by consolidation reduced-dose wholebrain radiotherapy and cytarabine in newly diagnosed primary CNS lymphoma: final results and long-term outcome. J Clin Oncol. 2013;31(31):3971-9.
24 Omuro A, Correa DD, DeAngelis LM, Moskowitz $\mathrm{CH}$, Matasar MJ, Kaley TJ, et al. RMPV followed by high-dose chemotherapy with TBC and autologous stem-cell transplant for newly diagnosed primary CNS lymphoma. Blood. 2015;125(9):1403-10.

25 Ferreri AJ, Cwynarski K, Pulczynski E, Ponzoni M, Deckert M, Politi LS, et al. Chemoimmunotherapy with methotrexate, cytarabine, thiotepa, and rituximab (MATRix regimen) in patients with primary CNS lymphoma: results of the first randomisation of the International Extranodal Lymphoma Study Group-32 (IELSG32) phase 2 trial. Lancet Haematol. 2016;3(5):e217-27.

26 Ferreri AJM, Cwynarski K, Pulczynski E, Fox CP, Schorb E, La Rosée P, et al. Whole-brain radiotherapy or autologous stem-cell transplantation as consolidation strategies after high-dose methotrexate-based chemoimmunotherapy in patients with primary CNS lymphoma: results of the second randomisation of the International Extranodal Lymphoma Study Group-32 phase 2 trial. Lancet Haematol. 2017;4(11):e510-23.

27 Correa DD, Maron L, Harder H, Klein M, Armstrong CL, Calabrese P, et al. Cognitive functions in primary central nervous system lymphoma: literature review and assessment guidelines. Ann Oncol. 2007;18(7):1145-51.

28 Correa DD, Shi W, Abrey LE, Deangelis LM, Omuro AM, Deutsch MB, et al. Cognitive functions in primary CNS lymphoma after single or combined modality regimens. Neuro-oncology. 2012;14(1):101-8.

29 Omuro A, MP, DeAngelis LM, Karrison T, Bovi J A, Rosenblum M, et al. Randomized phase II study of rituximab, methotrexate (MTX), procarbazine, vincristine, and cytarabine (R-MPV-A) with and without low-dose whole-brain radiotherapy (LD-WBRT) for newly diagnosed primary CNS lymphoma (PCNSL). J Clin Oncol. 2020;38(Suppl 15).

30 Batchelor T, Sharmila G, Ruppert AS, Bartlett NL, Hsi ED, Cheson BD, et al. Myeloablative versus non-myeloablative consolidative chemotherapy for newly diagnosed primary central nervous system lymphoma: results of induction therapy in Alliance 51101. J Clin Oncol. 2020;38(Suppl 15).

31 Houillier C, Taillandier L, Dureau S, Lamy T, Laadhari M, Chinot O, et al. Radiotherapy or autologous stem-cell transplantation for primary CNS lymphoma in patients 60 years of age and younger: results of the intergroup ANOCEF-GOELAMS randomized phase II PRECIS study. J Clin Oncol. 2019;37(10): $823-33$.

32 Hoang-Xuan K, Bessell E, Bromberg J, Hottinger AF, Preusser M, Rudà R, et al. Diagnosis and treatment of primary CNS lymphoma in immunocompetent patients: guidelines from the European Association for NeuroOncology. Lancet Oncol. 2015;16(7):e32232. 
33 Schlegel U, Korfel A. Is whole-brain radiotherapy still a standard treatment for primary central nervous system lymphomas? Curr Opin Neurol. 2018;31(6):733-9.

34 Abrey LE, DeAngelis LM, Yahalom J. Longterm survival in primary CNS lymphoma. J Clin Oncol. 1998;16(3):859-63.

35 Lai R, Abrey LE, Rosenblum MK, DeAngelis LM. Treatment-induced leukoencephalopathy in primary CNS lymphoma: a clinical and autopsy study. Neurology. 2004;62(3):451-6.

36 Omuro AM, Ben-Porat LS, Panageas KS, Kim AK, Correa DD, Yahalom J, et al. Delayed neurotoxicity in primary central nervous system lymphoma. Arch Neurol. 2005;62(10): 1595-600.

37 Fisher B, Seiferheld W, Schultz C, DeAngelis L, Nelson D, Schold SC, et al. Secondary analysis of Radiation Therapy Oncology Group study (RTOG) 9310: an intergroup phase II combined modality treatment of primary central nervous system lymphoma. J Neurooncol. 2005;74(2):201-5.

38 Houillier $\mathrm{C}$, Ghesquières $\mathrm{H}$, Chabrot $\mathrm{C}$, Soussain C, Ahle G, Choquet S, et al. Rituximab, methotrexate, procarbazine, vincristine and intensified cytarabine consolidation for primary central nervous system lymphoma (PCNSL) in the elderly: a LOC network study. J Neurooncol. 2017;133(2):315-20.

39 Birsen R, Willems L, Pallud J, Blanc E, Burroni B, Legoff M, et al. Efficacy and safety of high-dose etoposide cytarabine as consolidation following rituximab methotrexate temozolomide induction in newly diagnosed primary central nervous system lymphoma in immunocompetent patients. Haematologica. 2018;103(7):e296-9.

40 Abrey LE, Moskowitz CH, Mason WP, Crump M, Stewart D, Forsyth P, et al. Intensive methotrexate and cytarabine followed by high-dose chemotherapy with autologous stem-cell rescue in patients with newly diagnosed primary CNS lymphoma: an intent-totreat analysis. J Clin Oncol. 2003;21(22): 4151-6.

41 Illerhaus G, Marks R, Ihorst G, Guttenberger $R$, Ostertag C, Derigs G, et al. High-dose chemotherapy with autologous stem-cell transplantation and hyperfractionated radiotherapy as first-line treatment of primary CNS lymphoma. J Clin Oncol. 2006;24(24):386570.

42 Soussain C, Suzan F, Hoang-Xuan K, Cassoux N, Levy V, Azar N, et al. Results of intensive chemotherapy followed by hematopoietic stem-cell rescue in 22 patients with refractory or recurrent primary CNS lymphoma or intraocular lymphoma. J Clin Oncol. 2001; 19(3):742-9.

43 Duarte RF, Labopin M, Bader P, Basak GW, Bonini $\mathrm{C}$, Chabannon $\mathrm{C}$, et al. Indications for haematopoietic stem cell transplantation for haematological diseases, solid tumours and immune disorders: current practice in Europe, 2019. Bone Marrow Transplant. 2019; 54(10):1525-52.
44 Illerhaus G, Kasenda B, Ihorst G, Egerer G, Lamprecht M, Keller U, et al. High-dose chemotherapy with autologous haemopoietic stem cell transplantation for newly diagnosed primary CNS lymphoma: a prospective, single-arm, phase 2 trial. Lancet Haematol. 2016; 3(8):e388-97.

45 Kassam S, Chernucha E, O’Neill A, Hemmaway C, Cummins T, Montoto S, et al. High-dose chemotherapy and autologous stem cell transplantation for primary central nervous system lymphoma: a multi-centre retrospective analysis from the United Kingdom. Bone Marrow Transplant. 2017;52(9): 1268-72.

46 Sanders S, Chua N, Larouche JF, Owen C, Shafey M, Stewart DA. Outcomes of consecutively diagnosed primary central nervous system lymphoma patients using the alberta lymphoma clinical practice guideline incorporating thiotepa-busulfan conditioning for transplantation-eligible patients. Biol Blood Marrow Transplant. 2019;25(8):1505-10.

47 Schorb E, Kasenda B, Atta J, Kaun S, Morgner A, Hess G, et al. Prognosis of patients with primary central nervous system lymphoma after high-dose chemotherapy followed by autologous stem cell transplantation. Haematologica. 2013;98(5):765-70.

48 DeFilipp Z, Li S, El-Jawahri A, Armand P, Nayak L, Wang N, et al. High-dose chemotherapy with thiotepa, busulfan, and cyclophosphamide and autologous stem cell transplantation for patients with primary central nervous system lymphoma in first complete remission. Cancer. 2017;123(16):3073-9.

49 Hyung J, Hong JY, Yoon DH, Kim S, Park JS, Park CS, et al. Thiotepa, busulfan, and cyclophosphamide or busulfan, cyclophosphamide, and etoposide high-dose chemotherapy followed by autologous stem cell transplantation for consolidation of primary central nervous system lymphoma. Ann Hematol. 2019; 98(7):1657-64.

50 Omuro A, Chinot O, Taillandier L, Ghesquieres H, Soussain C, Delwail V, et al. Methotrexate and temozolomide versus methotrexate, procarbazine, vincristine, and cytarabine for primary CNS lymphoma in an elderly population: an intergroup ANOCEFGOELAMS randomised phase 2 trial. Lancet Haematol. 2015;2(6):e251-9.

51 Schorb E, Finke J, Ihorst G, Kasenda B, Fricker H, Illerhaus G. Age-adjusted high-dose chemotherapy and autologous stem cell transplant in elderly and fit primary CNS lymphoma patients. BMC Cancer. 2019; 19(1):287.

52 Houillier C, Soussain C, Ghesquières H, Soubeyran P, Chinot O, Taillandier L, et al. Management and outcome of primary CNS lymphoma in the modern era: an LOC network study. Neurology. 2020;94(10):e102739.
53 Schorb E, Fox CP, Kasenda B, Linton K, Martinez-Calle $\mathrm{N}$, Calimeri $\mathrm{T}$, et al. Induction therapy with the MATRix regimen in patients with newly diagnosed primary diffuse large Bcell lymphoma of the central nervous system: an international study of feasibility and efficacy in routine clinical practice. Br J Haematol. 2020 Jun;185(5):879-87.

54 Martinez-Calle N, Poynton E, Alchawaf A, Kassam S, Horan M, Rafferty M, et al. Outcomes of older patients with primary central nervous system lymphoma treated in routine clinical practice in the UK: methotrexate dose intensity correlates with response and survival. Br J Haematol. 2020 Aug;190(3):394-404.

55 Abrey LE, Batchelor TT, Ferreri AJ, Gospodarowicz M, Pulczynski EJ, Zucca E, et al. International Primary CNSLCG: report of an international workshop to standardize baseline evaluation and response criteria for primary CNS lymphoma. J Clin Oncol. 2005;23:5034-43.

56 Miyakita Y, Ohno M, Takahashi M, Kurihara $\mathrm{H}$, Katai H, Narita Y. Usefulness of carbon11-labeled methionine positron-emission tomography for assessing the treatment response of primary central nervous system lymphoma. Jpn J Clin Oncol. 2020;50(5): 512-8.

57 Duarte RF, Sanchez-Ortega I. HSCT in elderly patients. In: Carreras E, Dufour C, Mohty M, Kroger N, editors. The EBMT handbook: hematopoietic stem cell transplantation and cellular therapies. Cham; 2019. p. 499-503.

58 Schorb E, Fox CP, Fritsch K, Isbell L, Neubauer A, Tzalavras A, et al. High-dose thiotepa-based chemotherapy with autologous stem cell support in elderly patients with primary central nervous system lymphoma: a European retrospective study. Bone Marrow Transplant. 2017;52(8):1113-9.

59 Colombat P, Lemevel A, Bertrand P, Delwail V, Rachieru P, Brion A, et al. High-dose chemotherapy with autologous stem cell transplantation as first-line therapy for primary CNS lymphoma in patients younger than 60 years: a multicenter phase II study of the GOELAMS group. Bone Marrow Transplant. 2006;38(6):417-20.

60 Wiebe VJ, Smith BR, DeGregorio MW, Rappeport JM. Pharmacology of agents used in bone marrow transplant conditioning regimens. Crit Rev Oncol Hematol. 1992;13(3): 241-70.

61 Makale MT, McDonald CR, Hattangadi-Gluth JA, Kesari S. Mechanisms of radiotherapyassociated cognitive disability in patients with brain tumours. Nat Rev Neurol. 2017;13(1): 52-64.

62 Pazzaglia S, Briganti G, Mancuso M, Saran A Neurocognitive decline following radiotherapy: mechanisms and therapeutic implications. Cancers. 2020;12(1):146.

63 Cascella M, Di Napoli R, Carbone D, Cuomo GF, Bimonte S, Muzio MR. Chemotherapyrelated cognitive impairment: mechanisms, clinical features and research perspectives. Recenti Prog Med. 2018;109(11):523-30. 
64 Walczak P, Janowski M. Chemobrain as a product of growing success in chemotherapy: focus on glia as both a victim and a cure. Neuropsychiatry. 2019;9(1):2207-16.

65 Berlin C, Lange K, Lekaye HC, Hopland K, Phillips S, Piao J, et al. Long-term clinically relevant rodent model of methotrexate-induced cognitive impairment. Neuro Oncol. 2020 Aug 17;22(8):1126-37.

66 Bhatia S, Robison LL, Francisco L, Carter A, Liu Y, Grant M, et al. Late mortality in survivors of autologous hematopoietic-cell transplantation: report from the Bone Marrow Transplant Survivor Study. Blood. 2005; 105(11):4215-22.

67 Kelly DL, Buchbinder D, Duarte RF, Auletta JJ, Bhatt N, Byrne M, et al. Neurocognitive dysfunction in hematopoietic cell transplant recipients: expert review from the late effects and Quality of Life Working Committee of the Center for international blood and marrow transplant research and complications and Quality of Life Working Party of the European Society for blood and marrow transplantation. Biol Blood Marrow Transplant. 2018;24(2):228-41.

68 Buchbinder D, Kelly DL, Duarte RF, Auletta JJ, Bhatt N, Byrne M, et al. Neurocognitive dysfunction in hematopoietic cell transplant recipients: expert review from the late effects and Quality of Life Working Committee of the CIBMTR and complications and Quality of Life Working Party of the EBMT. Bone Marrow Transplant. 2018;53(5):535-55.

69 Correa DD, Braun E, Kryza-Lacombe M, Ho KW, Reiner AS, Panageas KS, et al. Longitudinal cognitive assessment in patients with primary CNS lymphoma treated with induc- tion chemotherapy followed by reduced-dose whole-brain radiotherapy or autologous stem cell transplantation. J Neurooncol. 2019; 144(3):553-62.

70 Harder H, Cornelissen JJ, Van Gool AR, Duivenvoorden HJ, Eijkenboom WM, van den Bent MJ. Cognitive functioning and quality of life in long-term adult survivors of bone marrow transplantation. Cancer. 2002;95(1): 183-92.

71 Harder H, Duivenvoorden HJ, van Gool AR, Cornelissen JJ, van den Bent MJ. Neurocognitive functions and quality of life in haematological patients receiving haematopoietic stem cell grafts: a one-year follow-up pilot study. J Clin Exp Neuropsychol. 2006;28(3):283-93.

72 Abrey LE, Yahalom J, DeAngelis LM. Treatment for primary CNS lymphoma: the next step. J Clin Oncol. 2000;18(17):3144-50. 